\title{
READMISSIONS TO HOSPITAL: AN INDICATOR OF QUALITY OF CARE BEFORE AND DURING THE WAITING LIST REDUCTION PROGRAM
}

Kathy Jong, NSW Department of Health

Paul Corben, Mid North Coast Population Health Unit, Port Macquarie

The waiting list reduction program was introduced to improve access to elective surgery in public hospitals throughout NSW. The main aim of the the program was to halve the number of patients awaiting admission for elective surgery within the first 12 months, with particular emphasis on reducing the number of patients waiting more than six months. There is evidence that the program was successful in reducing elective surgery waiting lists and waiting times ${ }^{1}$. This article examines a possible indicator of the quality of care of elective patients in public hospitals, before and after the introduction of the program.

There are no agreed methods of assessing the quality of clinical care. One proposed indicator which is widely used comprises unplanned or 'emergency' readmissions to hospital, which may reflect adverse outcomes of initial hospitalisation. This analysis examined emergency readmissions within 28 days of discharge, where the admission and readmission involved the same hospital. The analysis took account of the types of hospital and patient involved, and covered elective medical and surgical admissions.

\section{METHODS}

\section{Data source}

Data were obtained from the NSW Inpatient Statistics Collection. This provides information on all discharges and transfers from all NSW public and private hospitals, as well as deaths in hospital. Data on discharges during two sixmonth periods (July-December 1994 and July-December 1995) were compared. At the time of analysis, data for the latter period were incomplete, and this may have resulted in an underestimate of 1995 readmission rates.

\section{Calculation of readmission rates}

For each six-month period, readmission rates were calculated by:
1. determining the number of first emergency readmissions within 28 days of discharge or transfer after an elective episode of care;
$2 . \quad$ determining the number of discharges or transfers following elective admissions; and 3. dividing 1 . by 2 .

Patients admitted repeatedly for continuing renal dialysis or chemotherapy were not counted as emergency readmissions, and patients who died during elective admissions were excluded.

As we were interested in elective admissions completed up to and including December 31 of each of the two years studied (1994 and 1995), it was necessary to identify readmissions beginning up to and including January 28 in each following year (1995 and 1996). Hospitals were placed into seven categories, according to the types of services they provide ${ }^{2}$ :

1. $\quad$ public principal referral hospitals: large teaching hospitals providing high-level specialty services, located in metropolitan areas;
2. major public referral hospitals: either teaching or emerging teaching hospitals providing a less extensive range of specialty services than principal referral hospitals;

3. major rural base hospitals: hospitals providing a wide range of specialist services and functioning as referral hospitals for defined populations outside metropolitan areas.

District public hospitals provide a wide range of services to local populations with some subspecialty services. To enable comparison, these hospitals are subgrouped according to size, level of service provision and the range and type of patients in the hospital. These categories include:

4. district (high)

$5 . \quad$ district (medium)

6. $\quad$ district (low)

7. community public: hospitals providing basic routine and emergency health care for local populations. These hospitals may also provide nursing home-type care.

An emergency readmission may be the result of several factors, including the nature of the preceding elective admission and the condition of the patient. For example, an older patient with diabetes admitted for coronary artery surgery is more likely to have an emergency readmission than a young adult admitted for arthroscopy.

To help in the interpretation of readmission rates, patients were grouped according to the nature of their elective admission (classified into diagnosis-related groups (ANDRGs), five-year age groups, and sex, and also according to the hospital type. Within each hospital type, a readmission rate was calculated for each ANDRG, age and sex group in July-December 1994. These rates were taken as baseline rates. The numbers of patients in each hospital type, ANDRG, age and sex group in 1994 and 1995 were multiplied by these baseline rates, and a summary rate for each hospital type in 1994 and 1995 was calculated. Through this process (known as indirect standardisation) the summary rates for 1994 and 1995 were comparable because differences in the types of elective admissions and patient characteristics between the two periods had been taken into account.

This procedure was repeated for 'medical' and 'surgical' elective admissions separately (according to a classification of admission types developed by Dr Paul Tridgell, Evaluation and Monitoring Branch, NSW Health Department).

Statistical analysis

For each type of hospital and for the totals across all types of hospitals combined, statistical tests of the difference between proportions were used to determine whether the rates (expressed as a percentage) were the same in the two periods ${ }^{3}$. The results, including 95 per cent confidence intervals (CIs) for the rates, are shown in Figures 1 and 2 and Tables 4 and 5. 


\section{Readmissions to hospital}

\section{Continued from page 33}

\section{RESULTS}

The unplanned-readmission rate after elective admission showed no significant change between the six-month periods in 1994 and 1995. Adjusted readmission rates were 2.3 per cent in 1994 and 2.2 per cent in 1995.

\section{Elective surgical patients (Figure 1)}

For elective surgical patients there was no significant change in the unplanned readmission rate between the two periods. Trends in readmission rates varied among the different types of hospital (Table 1).

\section{Elective medical patients (Figure 2)}

For elective medical patients there was a significant reduction in the unplanned readmission rate from 2.2 per cent to 2.0 per cent (Table 2). Again, there was variation in the readmission rates among the different hospital types.

\section{CONCLUSION}

According to this analysis there was no consistent change in the average rates of emergency readmissions for elective patients in NSW hospitals between 1994 and 1995. While readmissions after elective medical admission decreased, no significant change was detected after elective surgical admissions.

Emergency readmission rates decreased significantly between 1994 and 1995 for elective surgical and medical patients in the principal referral group of hospitals. However, there were significant increases in emergency readmission rates both for medical and surgical patients in the community public hospital group. Readmission rates decreased significantly for elective medical patients but increased significantly for elective surgical patients in the major referral hospital group.

Our analysis took account of age, sex and ANDRG (version 3 ) in adjusting for the risk of emergency readmission to hospital. While we would not expect substantial changes in hospital caseload from year to year, it is possible that the DRG system does not adequately account for differences in severity of illness, or other factors which may affect readmission to hospital. Consequently, the fluctuation in readmission rates which we found may have been due to changes in other characteristics of patients as well as random (or chance) variation from one year to the next.

Although the apparent plausibility (face validity) of emergency admission rates is generally accepted, rigorous study is needed to validate readmission rates as an indicator of quality of care. A recent meta-analysis of 16 studies that examined quality of care based on descriptions of the process of care and early readmission rates indicates that care of a relatively low quality increases the odds of readmission by 55 per cent, compared with care of a higher quality (95 per cent CI 1.25 to 1.92). Analysis of the studies that specifically described elements of substandard inpatient care found that, compared with normative care, it increased the odds of readmission by 24 per cent ( 95 per cent CI 0.99 to 1.57). Only 11 comparisons contributed to this latter analysis, and the wide confidence interval (which includes 1.0) and heterogeneity among the studies indicates that the results should be interpreted cautiously.

\section{FIGURE 1}

ELECTIVE SURGICAL PATIENTS, JULY-DECEMBER 1994 AND JULYDECEMBER 1995: RATES PER 100 OF EMERGENCY READMISSION WITHIN 28 DAYS (AND 95\% CONFIDENCE INTERVALS), STANDARDISED FOR ANDRG, AGE GROUP AND SEX

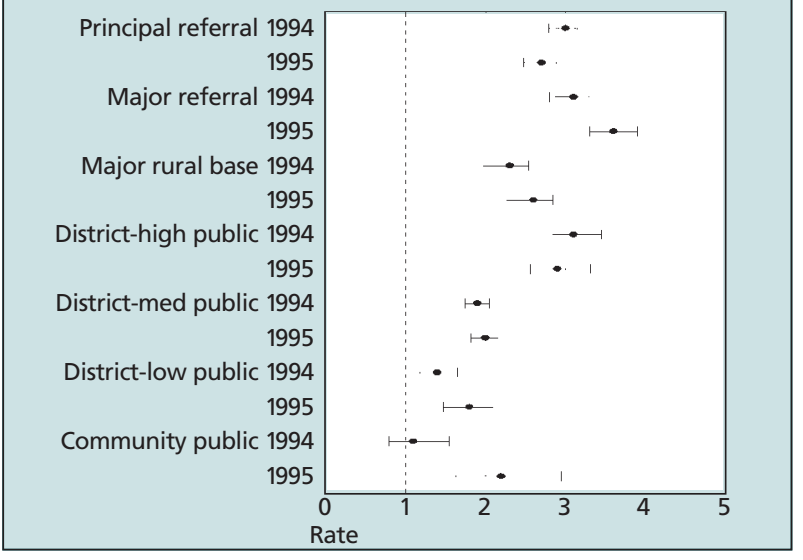

\section{FIGURE 2}

ELECTIVE MEDICAL PATIENTS, JULY-DECEMBER 1994 AND JULYDECEMBER 1995: RATES PER 100 OF EMERGENCY READMISSION WITHIN 28 DAYS (AND 95\% CONFIDENCE INTERVALS), STANDARDISED FOR ANDRG, AGE GROUP AND SEX

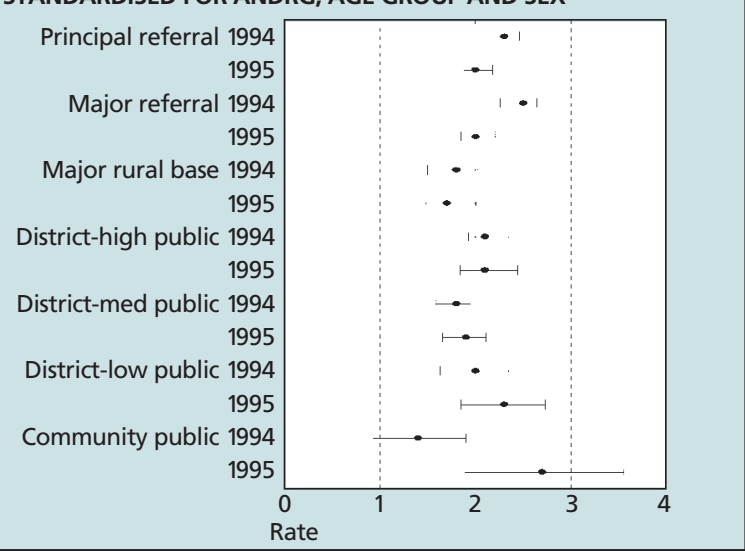

Epidemiologists at Monash University have recently evaluated this indicator as part of the National Validity \& Reliability Study. Their final report is being reviewed by the Commonwealth Department of Health and Family Services ${ }^{5}$.

Apart from questions of the validity of this indicator and the limitations of the routinely collected data on which this analysis has been based, other factors operating at the time may have influenced readmission rates in this analysis. Although the observed changes in readmission rates cannot necessarily be attributed to the waiting list reduction program, the methods used here serve as a prototype for further examination of unplanned readmission rates. 


\section{TABLE 4}

STANDARDISED READMISSION RATES (\%) FOR ELECTIVE SURGICAL ADMISSIONS: JULY-DECEMBER 1994 AND JULY-DECEMBER 1995

\begin{tabular}{|c|c|c|c|c|}
\hline Hospital role grouping & $\begin{array}{c}\text { Emergency } \\
\text { readmission rate: } \\
1994 \\
\%\end{array}$ & $\begin{array}{c}\text { Emergency } \\
\text { readmission rate: } \\
1995 \\
\%\end{array}$ & $Z^{a}$ & $P$ \\
\hline 1. Public principal referral & 3.0 & 2.7 & 2.00 & $<0.05 \downarrow \downarrow$ \\
\hline 2. Public major referral & 3.1 & 3.6 & 2.51 & $<0.05 \uparrow$ \\
\hline 3. Major rural base & 2.3 & 2.6 & 1.49 & 0.14 \\
\hline 4. District high & 3.1 & 2.9 & 0.81 & 0.42 \\
\hline 5. District medium & 1.9 & 2.0 & 0.77 & 0.443 \\
\hline 6. District low & 1.4 & 1.8 & 1.96 & $<0.05 \uparrow$ \\
\hline 7. Community public & 1.1 & 2.2 & 3.00 & $<0.01 \uparrow \uparrow$ \\
\hline Total & 2.5 & 2.6 & 1.27 & 0.17 \\
\hline $\begin{array}{l}\text { Notes: } \\
\text { (a) Using normal approximation } \\
\text { (b) } \downarrow=\text { statistically significant de } \\
\text { (c) } \uparrow=\text { statistically significant inc }\end{array}$ & & & & \\
\hline
\end{tabular}

\section{TABLE 5}

STANDARDISED READMISSION RATES (\%) FOR ELECTIVE MEDICAL ADMISSIONS: JULY-DECEMBER 1994 AND JULY-DECEMBER 1995

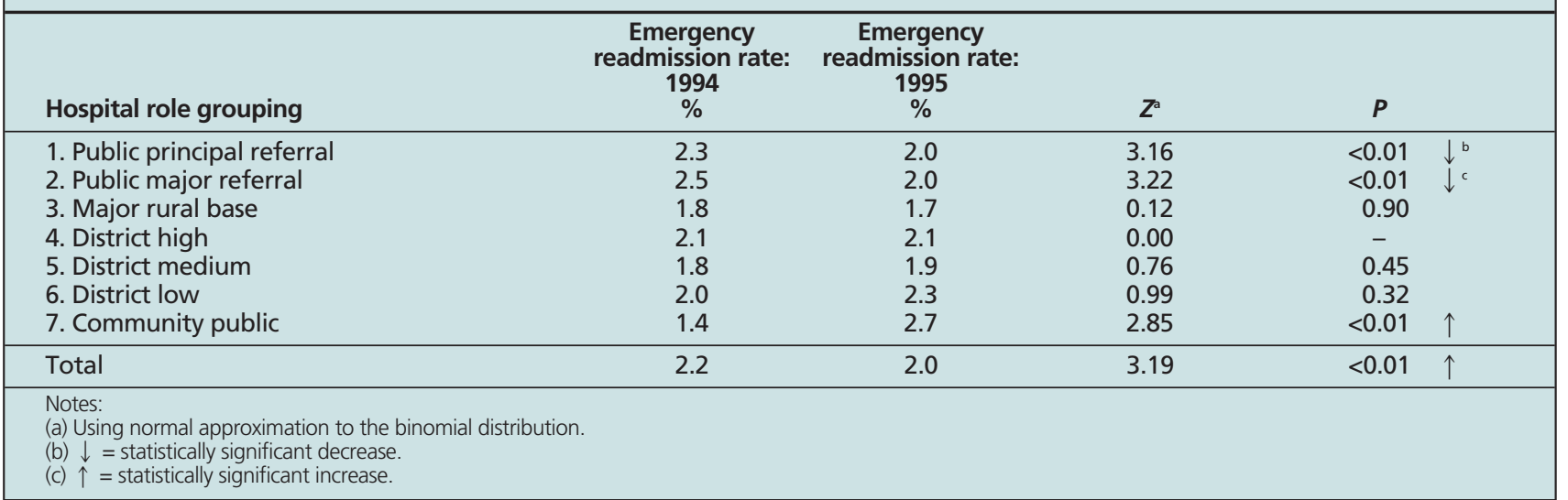

1. Caring for health: after the waiting list reduction program: continuous improvement in patient management. State Health pubn no. 96-0016. Sydney: NSW Department of Health, 1996.

2. NSW public hospitals comparison data 1994-95. State Health pubn no. (IDS) 96-0068. Sydney: NSW Department of Health, 1996.

3. Kirkwood BR. Essentials of medical statistics. Oxford: Blackwell Scientific, 1988.

4. Ashton, CM, Del Junco DJ, Souchek J, Wray NP, Mansyur CL. The

association between the quality of inpatient care and early readmissions:

a meta analysis of the evidence. Med Care 1997; 35: 1044-59.

5. McNeill JJ, Boyce N, de Campo M, Ibrahim J, Majoor J. Literature

review: reliability and validity and risk adjustment of a pilot set of

hospital wide indicators. Draft report. Canberra: Department of Human

Services and Health, 1996. 